\title{
Vanishing lung syndrome in one family: Five cases with a 20-year follow-up
}

\author{
XICHUN GAO ${ }^{1}$, HAIYING WANG ${ }^{2}$, KAIHONG GOU ${ }^{3}$, BAOSHENG HUANG ${ }^{1}$, DONGZHOU XIA ${ }^{1}$, \\ XIULI WU ${ }^{1}$, MING WEI ${ }^{1}$, SHENGXI ZHENG ${ }^{1}$, SHAN MA ${ }^{1}$ and JUANXIANG HE ${ }^{1}$ \\ Departments of ${ }^{1}$ Radiology, ${ }^{2}$ Medicine and ${ }^{3}$ Obstetrics and Gynecology, The People's Hospital of Zhangye Municipality, \\ Zhangye, Gansu 734000, P.R. China
}

Received January 19, 2014; Accepted July 31, 2014

DOI: $10.3892 / \mathrm{mmr} .2014 .2673$

\begin{abstract}
Vanishing lung syndrome, also known as idiopathic giant bullous emphysema, is a rare disease characterized by giant emphysematous bullae. The disease is diagnosed by radiological findings of giant bullae in one, or both, of the upper lobes of the lung, occupying at least one-third of the hemithorax. There have been several reports of vanishing lung syndrome, however it remains to be determined whether genetic inheritance is associated with the disease. In the present study, five patients within one family, with vanishing lung syndrome, were reported during a follow-up period of $\sim 20$ years. All of the patients were diagnosed by radiological findings, which showed diffuse bullae in the lungs, which were of varying size and asymmetrical distribution, and the occurrence of pneumothorax or emphysema. The Medical Ethics Committee of the People's Hospital of Zhangye Municipality (Zhangye, China) approved this study, and all subjects gave their informed consent During the follow-up period of 20 years, bullae in these patients were shown to progressively increase, and no other pulmonary diseases, including lung cancer, tuberculosis, pneumoconiosis and chronic bronchitis were observed. Autosomal dominant inheritance was observed in five cases, and autosomal recessive inheritance was observed in one case. The present study suggests that vanishing lung syndrome may be associated with autosomal dominant and recessive genetic inheritance.
\end{abstract}

\section{Introduction}

Vanishing lung syndrome, also known as idiopathic giant bullous emphysema, is a rare disease which is characterized by giant emphysematous bullae. In 1937, Burke (1) described

Correspondence to: Dr Xichun Gao, Department of Radiology, The People's Hospital of Zhangye Municipality, 67 Ring Road, Zhangye, Gansu 734000, P.R. China

E-mail: xichungao@outlook.com

Key words: vanishing lung syndrome, giant bullous emphysema, inheritance, high-resolution computed tomography a case of 'vanishing lungs' in a 35 year-old male, who had presented with progressive dyspnea, respiratory failure, and radiological and pathological findings of giant bullae. The radiological criteria for vanishing lung syndrome includes the presence of giant bullae in one or both of the upper lobes of the lung, occupying at least one-third of the hemithorax and compressing the surrounding normal lung tissue (2). There have been numerous reports of vanishing lung syndrome (3-7), however there are currently no case reports of vanishing lung syndrome within one family. In the present study, five patients with vanishing lung syndrome within one family, were reported and followed up for $\sim 20$ years.

\section{Case report}

Six members of three generations, in one Chinese family, were diagnosed with vanishing lung syndrome. One patient died of pulmonary heart disease with giant bullae and emphysema 29 years ago. The remaining five patients (one male and four females) were included in the present study, and followed up for 20 years. The average age of the patients was 54 years-old (range, 44-64 years), and the mean disease duration ranged between 12 and 50 years. All of the patients were diagnosed with vanishing lung syndrome according to the radiological criteria previously described by Roberts et al (2). None of the patients had any prior history of smoking. The major clinical characteristics of the patients are shown in Table 1. Autosomal dominant inheritance was observed in five cases, and autosomal recessive inheritance was observed in one case (Fig. 1).

All five patients did not exhibit any symptoms, such as coughing or expectoration, and they did not have any history of pulmonary diseases other than spontaneous pneumothorax. During the episodes of spontaneous pneumothorax the patients presented with symptoms including cough, dyspnea and progressive respiratory difficulty. On physical examination, one patient had normal lung function, three patients had moderate lung injury and one patient had severe lung injury. The first onset of spontaneous pneumothorax was treated with closed thoracic drainage for 1-3 days, after which almost all of the affected lung tissues were shown to be completely re-inflated. All five patients had a history of recurrent pneumothorax. For subject II-14, spontaneous 
pneumothorax first occurred in the right lung, and recurrent pneumothorax occurred in the left lung, one month later. For the proband, subject II-7 (Fig. 1), spontaneous pneumothorax first occurred at the age of 42 years, and another episode resulted 10 years later. Following the first recurrent pneumothorax, the patient suffered from a spontaneous pneumothorax every 2-3 years. Patient II- 6 had no recurrent pneumothorax following the treatment for the first pneumothorax 20 years ago, however all of the other patients were hospitalized between March and August, 2001 for spontaneous pneumothorax. Patients II-14, III-4, and II-7 underwent thoracoscopic bullectomy. For patient II-7, bullae in the left lung were successfully removed by thoracoscopic bullectomy, however the bullae in the right lung was failed to be removed, due to the large number and deep location of the bullae. Open surgery was performed in order to resect the bullae in the right lung.

All members of the family underwent chest X-rays. The five patients routinely underwent radiological examinations, including annual high-resolution computed tomography (HRCT). The patients were followed up for $\sim 20$ years. All of the radiological images in the present study were analyzed by two experienced radiologists.

\section{Results}

In the present study the main radiological findings included diffuse bullae in the lungs of varying size and asymmetrical distribution, and the occurrence of pneumothorax or emphysema.

The morphology of the bullae varied. The chest X-rays showed round, oval and irregular hyperlucent areas in the thorax. The bullae were observed as having thin walls $(1-2 \mathrm{~mm})$. Air-fluid levels were observed in one patient. The HRCT scans demonstrated that subpleural bullae were long and round, or irregular in shape, and that some bullae were 'bubble-like' (Fig. 2A). Intraparenchymal bullae were observed to be round or oval in shape (Fig. 2A and B).

The size of the bullae ranged from $<2-18 \mathrm{~cm}$ in diameter. For all of the patients, the majority of the bullae were $3-6 \mathrm{~cm}$ in diameter. The bullae which were $<2 \mathrm{~cm}$ in diameter were difficult to identify on the chest X-rays, however they were clearly observed on the HRCT scans. Subpleural bullae were large but less frequent, whereas parenchymal bullae were relatively small, with a diameter of $<3 \mathrm{~cm}$, but numerous. The HRCT scan of patient II-7 showed numerous bullae ( $>20$ bullae) with various sizes (Fig. 2A).

Diffuse bullae were asymmetrically distributed in both of the lungs, with upper and middle lobe predominance. Three of the five patients demonstrated upper and middle lobe predominant bullae in both lungs, and the other two patients had upper and middle lobe predominant bullae in just one lung. Bullae were also observed in the lower lung lobes of all of the patients. Bullae occurred in the right lower lobe in three patients, in the left lower lobe in four patients and in both lower lobes in two patients. In addition, all of the patients had subpleural bullae, and four patients had intraparenchymal bullae.

HRCT scans showed that medium and giant bullae exhibited as a hyperlucent area without any obvious lung markings. One or multiple septa lines were observed in the giant bullae 


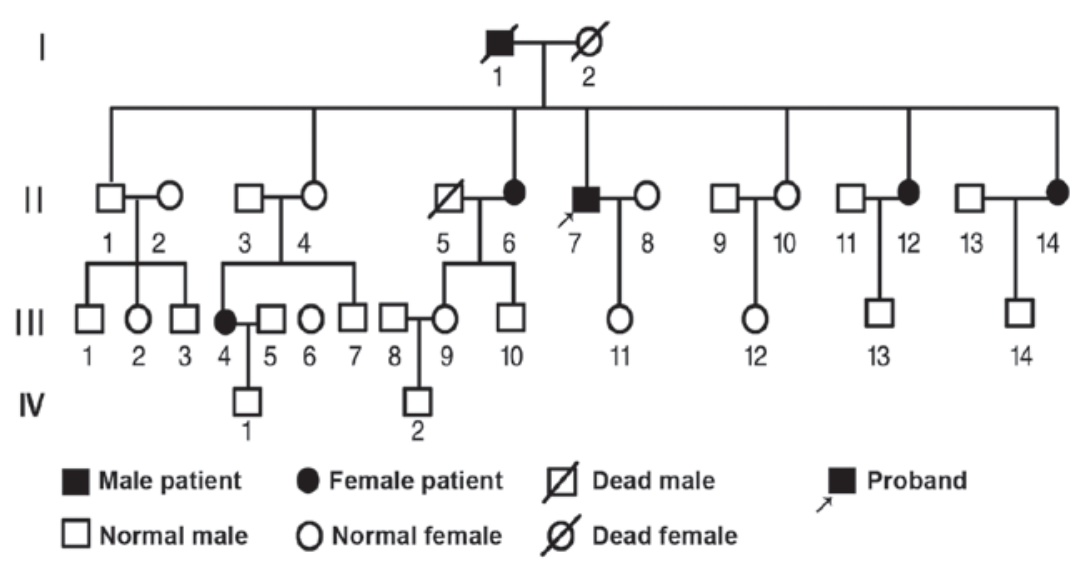

Figure 1. Genetic pedigree of the family with vanishing lung syndrome, reported within the present study.

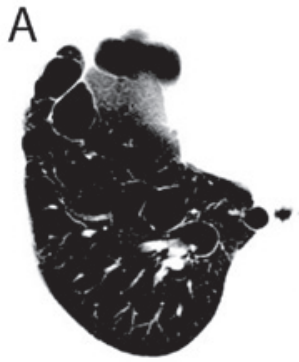

C

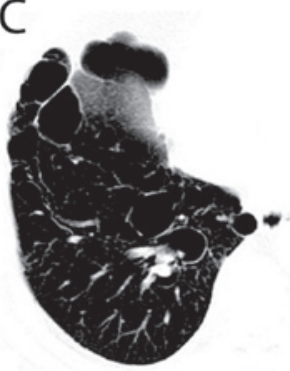

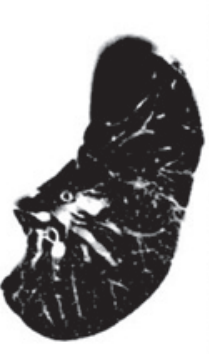
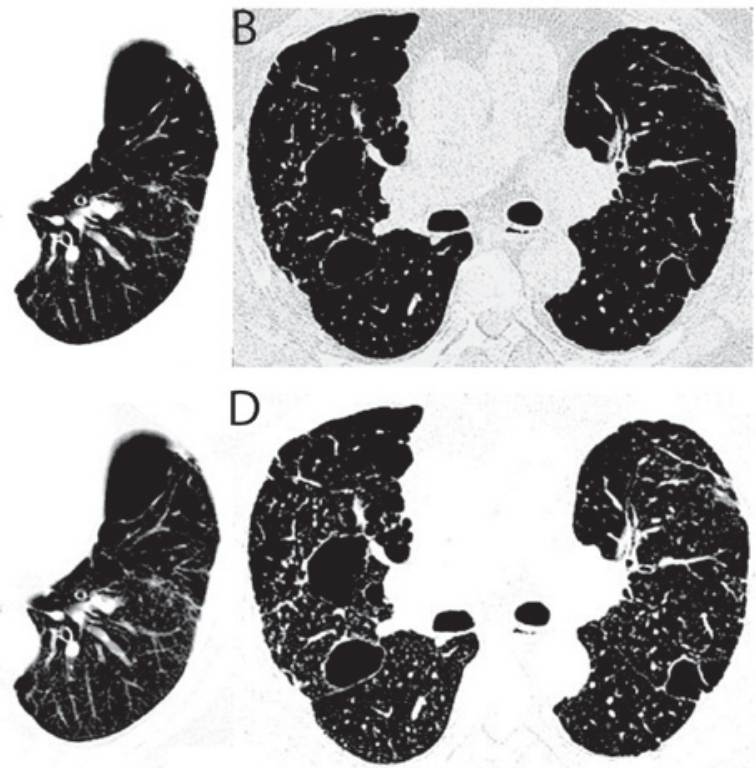

Figure 2. High-resolution computed tomography (HRCT) images from patients with vanishing lung syndrome within one family. (A) HRCT image showing subpleural bullae with irregular, or 'bubble-like' shapes of various sizes. (B) HRCT image showing the round or oval shape of intraparenchymal bullae. (C) HRCT image showing that the normal lung parenchyma around the bullae was compressed. (D) HRCT image showing paraseptal pneumothorax and centrilobular emphysema.

in three cases, and the normal lung parenchyma was shown to be compressed together (Fig. 2C). Furthermore, chest $\mathrm{X}$-rays showed varying degrees of pneumothorax at different time points of the disease, in all five patients. HRCT scans showed that pneumothorax occurred in four patients (Fig. 2D), whereas pneumothorax was only identified in three patients in the chest X-rays. In addition, four of the five patients had paraseptal emphysema, which was mainly located in the upper and middle lung fields. Centrilobular emphysema was observed in two cases (Fig. 2D).

All patients were routinely followed up annually. The number and size of bullae was shown to increase in a time-dependent manner. New bullae occurred in the re-inflated lungs of the patients, following closed thoracic drainage for the treatment of pneumothorax. No spontaneous regression of the bullae was observed. In addition, during the follow-up period of $\sim 20$ years, pulmonary diseases such as lung cancer, tuberculosis, pneumoconiosis, chronic bronchitis and other chronic lung diseases were not observed in any of the patients. One patient additionally suffered from esophageal cancer.

\section{Discussion}

Vanishing lung syndrome is an idiopathic disease characterized by the presence of giant bullae within the lungs, which are different from the secondary bullae caused by chronic bronchitis, tuberculosis and pneumoconiosis (8). The disease is diagnosed by radiological findings of giant bullae in one or both of the upper lobes of the lung, occupying at least one-third of the hemithorax (2). In the present study, five cases of vanishing lung syndrome were reported in one family, all of which met the diagnostic criteria (2). In addition, during the 20 year follow-up period, bullae in these patients were shown to progressively increase, and no lung cancer, tuberculosis, pneumoconiosis or chronic bronchitis were observed, 
implying that these patients suffered from idiopathic vanishing lung syndrome. It has been previously reported that vanishing lung syndrome predominantly afflicts young male smokers (9). However, in the present study four out of the five patients reported with vanishing lung syndrome in the family were female, and none of them had a prior history of smoking. Furthermore, although previous studies have reported several cases of vanishing lung syndrome (3-7), the present study is the first, to our knowledge, to report on the cases of five patients with vanishing lung syndrome, all within one family.

The clinical manifestation of vanishing lung syndrome is dependent on the size, range and type of bullae, as well as the presence of spontaneous pneumothorax. Frequently, patients with vanishing lung syndrome exhibit symptoms of decreased lung function, and have cough, dyspnea and progressive respiratory difficulty during a pneumothorax episode (10). In the present study, all five patients were observed as not having any symptoms or signs when no pneumothorax occurred, and presented with cough, dyspnea and progressive respiratory difficulty during the pneumothorax episodes. In addition, bullae can be classified into three types: Type I, single bullae in one or both lungs; Type II, multiple subpleural and parenchymal bullae with a size of 3-6 $\mathrm{cm}$ in diameter in both lung; and Type III, diffuse bullae with different sizes in both lungs (11).

The etiology and pathogenesis of vanishing lung syndrome remains to be elucidated. It has previously been reported that bullae may be caused by dilation of the alveolar walls, due to congenital dysplasia or the insufficiency of pulmonary elastic fibrous tissues (12). Ruptured bullae lead to the occurrence of pneumothorax. A deficiency in the $\alpha 1$-antitrypsin protein has been previously reported to be associated with familial spontaneous pneumothorax (7). In addition, Sharpe et al (13) reported that the human leukocyte antigen haplotypes A2 and B40 were associated with familial spontaneous pneumothorax. In the present study, autosomal dominant inheritance was observed in five cases of vanishing lung syndrome, and autosomal recessive inheritance was observed in one. Therefore, autosomal dominant and recessive genetic inheritance may be associated with vanishing lung syndrome.

The diagnosis of vanishing lung syndrome is based on radiological imaging. Routine chest fluoroscopy and radiography is an economical and fast method used to identify the disease. Routine computed tomography scans, particularly HRCT scans, can identify the distribution, morphology, number and size of the bullae, as well as the range and type of emphysema and pneumothorax present. HRCT is also helpful in preoperatively determining the size, type and extent of bullae and emphysema in patients prior to undergoing bullectomy or lung volume reduction surgery. In addition,
HRCT may be used to identify the extent of lung compression, therefore providing a radiological basis for the planning of future surgical procedures. Stern et al (7) reported nine cases of vanishing lung syndrome, which were examined by HRCT, and found that the bullae were predominantly located in the upper lobes, and six patients had bullae in the lower lobes. These findings are consistent with the present study, which found that bullae were predominantly located in the upper and middle lobes, with the involvement of the lower lobes.

In conclusion, the present study reported on the case of five patients with vanishing lung syndrome, within one family. Vanishing lung syndrome was shown to be associated with autosomal dominant and recessive genetic inheritance. It may therefore be hypothesized that the diagnosis of familial vanishing lung syndrome should include two relatives also with the disease, as well as meeting the radiological criteria previously described by Roberts et al (2). In addition, the disease should be distinguished from secondary bullae caused by pulmonary diseases and cysts.

\section{References}

1. Burke R: Vanishing lungs: A case report of bullous emphysema. Radiology 28: 367-371, 1937.

2. Roberts L, Putman CE, Chen JTT, Goodman LR and Ravin CE: Vanishing lung syndrome: upper lobe bullous pneumopathy. Rev Interam Radiol 12: 249-255, 1987.

3. Mohammad K, Siddiqui MF and Badiredd S: The vanishing lungs! Am J Respir Crit Care Med 187: 448, 2013.

4. Liang JJ, Wigle DA and Midthun DE: Vanishing lung syndrome (idiopathic giant bullous emphysema). Am J Med Sci: Mar 27, 2013 (Epub ahead of print).

5. Tsao YT and Lee SW: Vanishing lung syndrome. CMAJ 184: E977, 2012.

6. Sood N and Sood N: A rare case of vanishing lung syndrome. Case Rep Pulmonol 2011: 957463, 2011.

7. Stern EJ, Webb WR, Weinacker A and Müller NL: Idiopathic giant bullous emphysema (vanishing lung syndrome): imaging findings in nine patients. AJR Am J Roentgenol 162: 279-282, 1994.

8. Roberts L, Putman CE, Chen JTT, Goodman LR and Ravin CE: Vanishing lung syndrome: upper lobe bullous pneumopathy. Rev Interam Radiol 12: 249-255, 1987.

9. Sharma N, Justaniah AM, Kanne JP, Gurney JW and Mohammed TL: Vanishing lung syndrome (giant bullous emphysema): CT findings in 7 patients and a literature review. J Thorac Imaging 24: 227-230, 2009.

10. Stern EJ and Frank MS: CT of the lung in patients with pulmonary emphysema: diagnosis, quantification, and correlation with pathologic and physiologic findings. AJR Am J Roentgenol 162: 791-798, 1994.

11. Wood JR, Bellamy D, Child AH and Citron KM: Pulmonary disease in patients with Marfan syndrome. Thorax 39: 780-784, 1984.

12. Menconi GF, Melfi FM, Mussi A, Palla A, Ambrogi MC and Angeletti CA: Treatment by VATS of giant bullous emphysema: results. Eur J Cardiothorac Surg 13: 66-70, 1998.

13. Sharpe IK, Ahmad M and Braun W: Familial spontaneous pneumothorax and HLA antigens. Chest 78: 264-268, 1980. 